\title{
Technical aspects of MRI signal change quantification after gadolinium-based contrast agents' administration
}

\author{
Joana Ramalho a,b, Miguel Ramalho ${ }^{\mathrm{a}, \mathrm{c}, *}$, Mamdoh AlObaidy ${ }^{\mathrm{a}, \mathrm{d}}$, Richard C. Semelka ${ }^{\mathrm{a}}$ \\ a Department of Radiology, University of North Carolina, Chapel Hill, North Carolina, United States \\ b Department of Radiology, Centro Hospitalar de Lisboa Central, Lisbon, Portugal \\ c Department of Radiology, Hospital Garcia de Orta, Almada, Portugal \\ d Department of Radiology, King Faisal Specialist Hospital E Research Center, Riyadh. Saudi Arabia
}

\section{A R T I C L E I N F O}

\section{Article history:}

Received 21 July 2016

Accepted 21 September 2016

\section{Keywords:}

Sequences

Signal intensity

T1

Signal change

Gadolinium accumulation

\begin{abstract}
A B S T R A C T
Over the last 2 years several studies have been published regarding gadolinium deposition in brain structures in patients with normal renal function after repeated administrations of gadolinium-based contrast agents (GBCAs). Most of the publications are magnetic resonance imaging (MRI) based retrospective studies, where gadolinium deposition may be indirectly measured by evaluating changes in T1 signal intensity (SI) in brain tissue, particularly in the dentate nucleus (DN) and/or globus pallidi (GP). The direct correlation between $\mathrm{T} 1$ signal changes and gadolinium deposition was validated by human pathology studies. However, the variability of the MR equipment and parameters used across different publications, along with the inherent limitations of MRI to assess gadolinium in human tissues should be acknowledged when interpreting those studies. Nevertheless, MRI studies remain essential regarding gadolinium bio-distribution knowledge. The aim of this paper is to overview current knowledge of technical aspects of T1 signal intensity evaluation by MRI and describe confounding factors, with the intention to achieve higher accuracy and maximize reproducibility.
\end{abstract}

(C) 2016 Elsevier Inc. All rights reserved.

\section{Introduction}

In the past 2 years several magnetic imaging (MRI) studies reported high T1 signal intensity in the dentate nucleus (DN) and/or globus pallidi (GP) in patients with normal renal function after multiple administrations of gadolinium-based contrast agents (GBCAs) suggesting gadolinium deposition [1-9]. The agent most commonly implicated was gadodiamide $\left(\right.$ Omniscan $\left.^{\circledR}\right)$ a non-ionic linear agent, classified according to its stability as a weak agent [10], followed by gadopentetate dimeglumine (Magnevist ${ }^{\circledR}$ ) and gadobenate dimeglumine (MultiHance ${ }^{\circledR}$ ), both ionic linear agents, classified as agents with intermediate stability [10]. The more stable macrocyclic compounds: gadoteridol (ProHance ${ }^{\circledR}$ ), gadoterate meglumine (Dotarem ${ }^{\circledR}$ ), and gadobutrol (Gadavist ${ }^{\circledR}$ ) have not been associated with substantial MR imaging changes, suggesting that the molecular structure of the GBCA, whether linear or macrocyclic, is a crucial factor for the increase in signal intensity.

Gadolinium deposition in brain tissue and its relation to T1 signal intensity changes was confirmed by histopathological human studies

\footnotetext{
* Corresponding author.

E-mail addresses: Joana-Ramalho@netcabo.pt (J. Ramalho), Miguel-ramalho@netcabo.pt (M. Ramalho),mamdoh.alobaidy@yahoo.com (M. AlObaidy), richsem@med.unc.edu (R.C. Semelka).
}

[10-13]. However, these studies found that gadolinium deposits were present in all evaluated brain tissues (with higher concentration in DN followed by GP) after the administration of either linear or macrocyclic agents, suggesting that MRI has a relative limited sensitivity to detect gadolinium deposition in brain tissue. This limitation should have been expected, as bone deposition, which has been recognized for a decade [14], occurs at much higher levels compared to brain tissue [13], and yet is not detected by MRI. Despite limited sensitivity, MRI remains the best available tool to evaluate gadolinium deposition in basal ganglia, which appears to correlate well and proportionately with the higher deposition in bone [13].

Recent peer-reviewed papers have been published in high impact factor radiology journals and represent similar experience reported by experts from all over the world. Some critical methodological issues have not been adequately standardized in a number of these studies including the sequences used to evaluate T1 signal intensity changes overtime, whether signal measurements between sequences can be correlated, the ratios used and which are optimal, the potential effect of field strength; the reliability of excluding previous gadolinium expositions; number of doses and volume of doses administered, the diseases of subjects and possible differences of retention between different disease types, and the age range of the studied population. Thus, the aim of this review is to evaluate and recommend imaging strategies to improve data accuracy, and 
identify potential confounding factors in assessing gadolinium deposition in MRI-studies.

\section{MRI quantification of signal changes after (GBCAs) administration: basic principles}

One major limitation of MRI human studies reporting gadolinium deposition is its retrospective nature. This unavoidable study design explains in part the variability of the MR imaging protocols used, which may change according to the pathology that is being studied and among different institutions. Quantitative and qualitative analysis have been described. Quantitative signal intensity ratios are favored and recommended for scientific research; however, it is difficult to apply in clinical practice. Conversely qualitative/visual assessment analysis is applied every day to assess normal brain structures and lesions, but is less accurate and less appropriate for scientific studies. Regardless of the type of analysis some general guidelines should be followed. For clarity purposes, we will address each technical parameter and methodological aspect separately.

\section{Field strength and types of T1-weighted sequences}

The influence of MR field strength in quantitative and qualitative evaluation of T1 signal intensity of DN or other brain structures is presently unknown. It seems reasonable to assume that signal intensity will differ between $1.5 \mathrm{~T}$ and $3 \mathrm{~T}$, but so far no studies have addressed this question. Therefore, we suggest analyzing data obtained from different field strength scans separately, i.e., for a given patient, the first (baseline) MRI and subsequent MRIs should be performed on the same field strength for an accurate comparison.

In most of the published studies, the authors have used T1-weighted spin echo (SE) to evaluate the DN and/or GP signal intensity and signal changes over time. However, in some quantitative $[4,7,13]$, and qualitative $[13,15]$ studies, different T1-weighted sequences have been interchangeably used, including T1 SE, T1-weighted three-dimensional magnetization-prepared rapid gradient-echo (3D MPRAGE) [4], T1 FLASH (fast low-angle shot) [7], and T1 fluid attenuation inversion recovery (FLAIR) [15]. Recognizing this potential confounding factor, Radbruch et al. [4] performed a subgroup analysis to examine whether the two different T1-weighted sequences applied (MPRAGE and SE) had an influence on the signal intensity ratio difference. The authors found no statistically significant influence. In contrast, Ramalho et al. [16] performed an intra-individual qualitative and quantitative comparison between T1 SE and T1 3D MPRAGE in patients who had multiple exposures to gadodiamide (Omniscan ${ }^{\circledR}$ ) and found that both sequences cannot be used interchangeably for qualitative or quantitative analysis of signal intensity in the DN. They showed that baseline and final examination ratios should be evaluated over time by always using the same sequences. Also, they suggested that qualitative analysis is better performed with MPRAGE since its correlates better with quantitative analysis offering advantages over SE sequences for research purposes.

It should be recognized that it is virtually impossible to exclude some variability even when using the same imaging sequence, especially if performed on different machines and at different institutions. Significant variations in the sequence parameters and technique over time are expected to provide significant variation of the SI measurements. Nevertheless, despite these factors, variability is much lower if the same type of sequence is used consistently, compared with the use of completely different sequences. The aim should be to keep the sequence parameters as consistent as possible.

One interesting possibility that requires confirmation, is a study by Tanaka et al. [17] that examined whether noncontrast T1-weighted images could be replaced with T1-weighted images obtained following injection of gadolinium; which they did by comparing the signal intensity ratio of the DN-to-pons of the two sequences in multiple sclerosis (MS) patients in whom accumulation of gadolinium was previously seen. No significant difference was found between the two sequences, suggesting that the effects of long-term accumulation of gadolinium could be analyzed by examining T1-weighted images acquired after injection of gadolinium. This approach can be very useful in circumstances when the only available sequence for long-term analysis is the post-gadolinium T1-weighted sequence.

\section{Quantitative ratios measurements}

Different ratios have been used for quantitative measurements including DN-to-pons, DN-to-middle cerebellar peduncle, DN-to-cerebellar white matter, DN-to-CSF, DN-to-corpus callosum genu (CC), GP-to-Th, GP-to-CSF, GP-to-CC, Th-to-CC, and Caudate nucleus-to-CC [1-9,17-20]. In a study with healthy volunteers, T1 SI of DN, pons, GP, white matter, and gray matter were all evaluated and normalized with CSF [21].

Up to the present time, no dedicated study has focused on comparing these ratios in their entirety, although Radbruch et al. [4] compared DN T1 signal intensity with that of pons, CSF, and cerebellum. The authors found comparable results analyzing DN-to-pons, DN-to-CSF, and DN-to-cerebellum ratios over time in patients who received gadopentetate dimeglumine (Magnevist ${ }^{\circledR}$ ) or gadoterate meglumine (Dotarem ${ }^{\circledR}$ ).

Theoretically CSF should not be used as a control structure since it is believed that GBCAs are able to cross blood-CSF barrier. In 2001, Rai and Hogg [22] reported two dialysis-dependent patients with end-stage renal disease who showed increased signal intensity in the subarachnoid space on T1-weighted and FLAIR images after contrast enhanced MRI studies suggesting CSF gadolinium diffusion. Excretion of gadolinium into the CSF was proven in one case by mass spectrometry. Later, two case reports of presumed gadolinium induced encephalopathy with CSF T1 hyperintensity were published by Maramattom et al. [23] and Hui et al. [24]. All described studies reported patients with renal impairment. Recently, an animal study by Jost et al. [25] showed enhanced signal intensity of CSF spaces in the post-contrast FLAIR images of all animals receiving either linear (gadodiamide $\left(\right.$ Omniscan $\left.^{\circledR}\right)$, gadopentetate dimeglumine (Magnevist $\left.{ }^{\circledR}\right)$, and gadobenate dimeglumine (MultiHance $\left.{ }^{\circledR}\right)$ ) or macrocyclic (gadobutrol (Gadavist ${ }^{\circledR}$ ) and gadoterate meglumine (Dotarem ${ }^{\circledR}$ ) GBCAs, but not with saline (control group) [25] suggesting that either linear or macrocyclic GBCAs are able to pass the blood-CSF barrier in healthy rats to a certain, not yet quantified, extent. How gadolinium can enter brain with an intact blood-brain barrier is not yet known. One possible pathway for gadolinium entering the brain might be passage of GBCAs directly from blood into the cerebrospinal fluid (CSF). Recently, Cao et al. [26] reported on the renal function effect on gadolinium-related signal increase on unenhanced T1-MRI, and found a trend toward increased choroid plexus signal intensity after GBCA exposure, which also supports this hypothesis.

At this point, the consensus of experts is that DN and GP are the main locations for gadolinium deposition in brain tissue and both represent the preferred structures for T1 signal evaluation on MRI studies, particularly the DN. However, it is still uncertain what is the best structure to normalize these measurements, or if any is necessary. Further studies are needed to address this.

Tedechi et al. [27] recently described a different MRI approach that could obviate this limitation. The authors evaluated changes on $\mathrm{T} 1$ and $\mathrm{T} 2 *$ relaxometry of DN with respect to the number of previous administrations of GBCAs in relapsing-remitting multiple sclerosis (RR-MS) patients. They found that the number of previous GBCA administrations correlated with R1 relaxation rates of DN, while R2* values remain unaffected, suggesting that T1-shortening in these 
patients is related to the amount of gadolinium that had been administered. In fact, while standard qualitative or quantitative analysis have variations between subjects, time-points, and imaging centers; the measurement of relaxation rates can offer a more consistent and reliable tissue characterization, as they are intrinsically related to tissue microstructure and are not affected by variations in the signal intensity of images or by acquisition factors (e.g., variable RF homogeneity and coil sensitivity profile) [27-29]. MR relaxometry likely provides accurate and reproducible quantitative information of gadolinium accumulation in DN; however, it requires specific gradient echo sequences in development that are not at present available on standard MR systems, and hence are not performed as part of the brain MRI protocol.

\section{GBCA related concerns}

Essentially all reported studies describe the number of GBCA administrations in order to evaluate the increased T1 signal over time. Some authors suggested that 6 or more contrast enhanced MRI were needed to evaluate T1 signal changes over time [1,2], while others evaluate the effect of only one high dose GBCA [21]. Provided that the number of doses were all accounted for, including brain and other contrast enhanced MRI, the critical factor is that only one GBCA should be evaluated for each patient to determine the potential for deposition of each agent.

It is accepted, that neural gadolinium deposition is more associated with multiple administrations of the less stable GBCAs, but the effect of these agents on subsequent more stable GBCA administrations is undetermined. Ramalho et al. [30] evaluated the impact of previous administration of gadodiamide $\left(\right.$ Omniscan $\left.^{\circledR}\right)$ and neural tissue gadolinium deposition in patients who received gadobenate dimeglumine (MultiHance ${ }^{\circledR}$ ). Their findings suggested a carry over effect of prior gadodiamide in gadolinium deposition from subsequent gadobenate dimeglumine administration. In clinical practice, patients may undergo MRI with different gadolinium chelates for a variety of reasons, including imaging studies performed at different facilities, and change in MRI contrast agents used at the same institution (often reflecting a shift from less stable GBCAs to more stable GBCAs). Based on these findings, we suggest separate subgroup analysis of patients who have received only one agent, from patients with a known history of different GBCAs administrations, when evaluating specific gadolinium deposition related to any specific GBCA, as multiple agents likely have additive effects even if the current agent is macrocyclic.

\section{Population variability}

Population demographics and diagnosis vary among studies. Some critical inclusion and exclusion criteria have been used to define the investigated patient populations. First, patients with brain lesions in the deep cerebellar nuclei unrelated to GBCA administration were excluded from analysis. In addition, patient populations were controlled for pre-existing diseases that are known to affect SI in the dentate nucleus or globus pallidus such as Langerhans cell histiocytosis and multiple sclerosis. T1 W hyperintensity in the GP has been linked to many other conditions including Wilson disease, hepatic dysfunction, Rendu-Osler-Weber disease, manganese toxicity, total parenteral nutrition, hemodialysis, and neurofibromatosis type 1 (31); which were also exclusion criteria adopted for nearly all published studies.

It is also critical that studies regarding the effect of GBCA administration in the brain take into account any potential abnormalities in kidney function. Cao et al. [26] recently demonstrated that the dentate T1 signal increase occurring in hemodialysis patients was significantly higher compared with that of control subjects matched for age, sex, and GBCA exposure; suggesting that the longer exposure to GBCAs, due to longer dwell time, leads to a greater effect on the DN in renal failure patients. Fortunately, all published studies either used abnormal kidney function as an exclusionary factor in their analysis or conducted careful controlled for kidney function to ensure that any gadolinium deposition in the brain was not caused by deficient clearance of GBCAs by the kidneys [31]. Additionally, as a general guideline, we recommend to evaluate and compare groups of patients with similar diagnosis, since at this point, it is not clear what the contribution is of the underlying pathologic process on gadolinium retention and deposition.

Regarding age, pediatric patients should be treated as a distinct group for analysis, and one of particular concern. Brain development begins early in fetal life and continues well into adolescence with prefrontal, posterior parietal, and other high-order association areas being the last areas to undergo myelination [32]. There is a dramatic proliferation of synapses in the prefrontal cortex during early and mid-childhood, reaching numbers that exceed adult levels by twoor three-fold during puberty, which is then followed by a plateau phase with subsequent elimination and reorganization of prefrontal synaptic connections during adolescence [32]. This time period is a critical period of development and has been considered particularly vulnerable to toxin exposure [19,33]; and the potential for gadolinium retention and incorporation in neural tissue in this period is unknown. High T1 SI related to the number of GBCAs administration has been reported in different pediatric studies [18-20] after administration of linear GBCAs, but how this compares to the MRI signal change in adults is still unknown.

\section{MRI limitations}

As stated above, MRI does not detect all the gadolinium deposits present in human tissues following repeated administration of GBCA. To date, bone deposition, which is likely the largest repository for gadolinium, is not demonstrable with MRI, and only the DN and GP exhibit MR visible deposition in the brain despite the gadolinium presence in essentially all brain tissue. This limitation may result from low sensitivity to lower gadolinium concentration, but it could also be explained by the presence of gadolinium of unknown composition and environment, or both.

It is currently considered that insoluble gadolinium salts, such as phosphate, bicarbonate, and hydroxide, or soluble protein-bound gadolinium should not have a known effect on T1 shortening. As such, MR imaging significantly underestimates how much gadolinium may be retained in human tissues where it is identified on MRI, and is largely invisible in most tissues where it is present [34].

Gadolinium tissue measurements are also influenced by many factors. Preparation of brain tissue may remove the water-soluble contrast agent, leaving only gadolinium bound to substances other than the chelate, and this may lead to underestimation of any accumulation. Also, there is certainly a lower threshold level of gadolinium where hyperintensity can no longer be seen on MR images, but gadolinium bound to something, unlikely the chelate, can still be measured [35]. Inductively coupled plasma mass spectrometry (ICP-MS) is also a destructive technique in which the tissue sample is incinerated. This means that it is unable to detect the gadolinium species present (chelated, unchelated, and what unchelated may be bound to). Additionally, some tissues (notably bone) are difficult to process.

\section{Summary}

In most MRI studies evaluating potential gadolinium deposition in brain tissue, researchers evaluated the SI ratios between the DN and GP compared to control regions, usually the pons and thalamus, 
respectively. Retrospective in nature, the analyses were performed on whichever T1-weighted MRI sequences that were performed at each institution including T1 SE, 3D MPRAGE, FLAIR, and FLASH; which account for an undesirable variability among the results. The amount and type of GBCA used in each clinical population varied; as did the age and clinical diagnosis of the patients included in each study. Accounting for all these variables is advisable to design future MRI-based studies in order to reach conclusions that are more reliable and reproducible. It is prudent to keep the MR field strength constant and the T1 weighed sequence used for each patient. T1 DNand GP-SI changes should be favored, while CSF should probably be avoided as a normalizer structure. New approaches for T1 SI evaluation may be used in prospective future studies. The GBCAs should be evaluated individually, and previous administration of other GBCAs should be avoided. Patients should be grouped by age, either pediatric or adults, and by diagnosis. Nevertheless, even controlling for all possible methodological variables, it is clear that with currently available MR techniques, the great majority of deposited gadolinium remains not detectable on MRI.

\section{References}

[1] Kanda T, Ishii K, Kawaguchi H, Kitajima K, Takenaka D. High signal intensity in the dentate nucleus and Globus pallidus on unenhanced T1-weighted MR images: relationship with increasing cumulative dose of a gadolinium-based contrast material. Radiology 2014;270(3):834-41.

[2] Errante Y, Cirimele V, Mallio CA, Di Lazzaro V, Zobel BB, Quattrocchi CC. Progressive increase of $\mathrm{T} 1$ signal intensity of the dentate nucleus on unenhanced magnetic resonance images is associated with cumulative doses of intravenously administered gadodiamide in patients with normal renal function, suggesting dechelation. Invest Radiol 2014:49(10):685-90.

[3] Kanda T, Osawa M, Oba H, Toyoda K, Kotoku J, Haruyama T, et al. High signal intensity in dentate nucleus on unenhanced T1-weighted MR images: association with linear versus macrocyclic gadolinium chelate administration. Radiology 2015;275(3):803-9.

[4] Radbruch A, Weberling LD, Kieslich PJ, Eidel O, Burth S, Kickingereder P, et al. Gadolinium retention in the dentate nucleus and Globus pallidus is dependent on the class of contrast agent. Radiology 2015;275(3):783-91.

[5] Quattrocchi CC, Mallio CA, Errante Y, Cirimele V, Carideo L, Ax A, et al. Gadodiamide and dentate nucleus $\mathrm{T} 1$ hyperintensity in patients with meningioma evaluated by multiple follow-up contrast-enhanced magnetic resonance examinations with no systemic interval therapy. Invest Radiol 2015;50(7):470-2.

[6] Ramalho J, Castillo M, AlObaidy M, Nunes RH, Ramalho M, Dale BM, et al. High signal intensity in Globus pallidus and dentate nucleus on unenhanced T1weighted MR images: evaluation of two linear gadolinium-based contrast agents. Radiology 2015:150872.

[7] Weberling LD, Kieslich PJ, Kickingereder P, Wick W, Bendszus M, Schlemmer H-P, et al. Increased signal intensity in the dentate nucleus on unenhanced T1-weighted images after gadobenate dimeglumine administration. Invest Radiol 2015:1.

[8] Radbruch A, Weberling LD, Kieslich PJ, Hepp J, Kickingereder P, Wick W, et al. High-signal intensity in the dentate nucleus and Globus pallidus on unenhanced T1-weighted images. Invest Radiol 2015;50(12):805-10.

[9] Cao Y, Huang DQ, Shih G, Prince MR. Signal change in the dentate nucleus on T1weighted MR images after multiple administrations of gadopentetate dimeglumine versus gadobutrol. Am J Roentgenol 2016;206(2):414-9.

[10] Frenzel T, Lengsfeld P, Schirmer H, Hütter J. Stability of gadolinium-based magnetic resonance imaging contrast agents in human serum at $37 \mathrm{C}$. Invest Radiol 2008;12:817-28.

[11] McDonald RJ, McDonald JS, Kallmes DF, Jentoft ME, Murray DL, Thielen KR, et al. Intracranial gadolinium deposition after contrast-enhanced MR imaging. Radiology 2015;275(3):772-82.

[12] Kanda T, Fukusato T, Matsuda M, Toyoda K, Oba H, Kotoku J, et al. Gadoliniumbased contrast agent accumulates in the brain even in subjects without severe renal dysfunction: evaluation of autopsy brain specimens with inductively coupled plasma mass spectroscopy. Radiology 2015;276(1):228-32.

[13] Murata N, Gonzalez-Cuyar LF, Murata K, Fligner C, Dills R, Hippe D, et al. Macrocyclic and other non-group 1 gadolinium contrast agents deposit low levels of gadolinium in brain and bone tissue. Invest Radiol 2016:1.

[14] Gibby WA, Gibby KA, Gibby WA. Comparison of Gd DTPA-BMA (Omniscan) versus Gd HP-DO3A (ProHance) retention in human bone tissue by inductively coupled plasma atomic emission spectroscopy. Invest Radiol 2004;39(3): 138-42.

[15] Adin ME, Kleinberg L, Vaidya D, Zan E, Mirbagheri S, Yousem DM. Hyperintense dentate nuclei on T1-weighted MRI: relation to repeat gadolinium administration. Am J Neuroradiol 2015.

[16] Ramalho J, RAMALHO M, AlObaidy M, Nunes RH, Castillo M, Semelka RC. T1 signal-intensity increase in the dentate nucleus after multiple exposures to gadodiamide: Intraindividual comparison between 2 commonly used sequences. Am J Neuroradiol 2016.

[17] Tanaka M, Nakahara K, Kinoshita M. Increased signal intensity in the dentate nucleus of patients with multiple sclerosis in comparison with Neuromyelitis Optica Spectrum disorder after multiple doses of gadolinium contrast. Eur Neurol 2016:195-8

[18] Miller JH, Hu HH, Pokorney A, Cornejo P, Towbin R. MRI brain signal intensity changes of a child during the course of 35 gadolinium contrast examinations. Pediatrics 2015;136(6):e1637-40.

[19] Roberts DR, Holden KR. Progressive increase of T1 signal intensity in the dentate nucleus and globus pallidus on unenhanced T1-weighted MR images in the pediatric brain exposed to multiple doses of gadolinium contrast. Brain and development. The Japanese Society of Child. Neurology 2016;38(3):331-6.

[20] $\mathrm{Hu} \mathrm{HH}$, Pokorney A, Towbin RB, Miller JH. Increased signal intensities in the dentate nucleus and globus pallidus on unenhanced T1-weighted images: evidence in children undergoing multiple gadolinium MRI exams. Pediatr Radiol 2016:1-9.

[21] Kromrey M-L, Liedtke KR, Ittermann T, Langner S, Kirsch M, Weitschies W, et al. Intravenous injection of gadobutrol in an epidemiological study group did not lead to a difference in relative signal intensities of certain brain structures after 5 years. Eur Radiol. Eur Radiol 2016:1-6.

[22] Rai AT, Hogg JP. Persistence of gadolinium in CSF: a diagnostic pitfall in patients with end-stage renal disease. AJNR Am J Neuroradiol 2001;22(7):1357-61.

[23] Maramattom BV, Manno EM, Wijdicks EFM, Lindell EP. Gadolinium encephalopathy in a patient with renal failure. Neurology 2005;64(7):1276-8.

[24] Hui FK, Mullins M. Persistence of gadolinium contrast enhancement in CSF: a possible harbinger of gadolinium neurotoxicity? Am J Neuroradiol 2008;30(1): e1.

[25] Jost G, Lenhard DC, Sieber MA, Lohrke J, Frenzel T, Pietsch H. Signal increase on unenhanced T1-weighted images in the rat brain after repeated, extended doses of gadolinium-based contrast agents. Invest Radiol 2016;51(2):83-9

[26] Cao Y, Zhang Y, Shih G, Zhang Y, Bohmart A, Hecht EM, et al. Effect of renal function on gadolinium-related signal increases on unenhanced T1-weighted brain magnetic resonance imaging. Invest Radiol 2016:1.

[27] Tedeschi E, Palma G, Canna A, Cocozza S, Russo C, Borrelli P, et al. In vivo dentate nucleus MRI relaxometry correlates with previous administration of gadolinium-based contrast agents. Eur Radiol 2016:1-8.

[28] Cheng H-LM, Stikov N, Ghugre NR, Wright GA. Practical medical applications of quantitative MR relaxometry. J Magn Reson Imaging 2012;36(4):805-24.

[29] Deoni SCL. Quantitative relaxometry of the brain. Top Magn Reson Imaging 2010;21(2):101-13.

[30] Ramalho J, Semelka RC, AlObaidy M, Ramalho M, Nunes RH, Castillo M. Signal intensity change on unenhanced T1-weighted images in dentate nucleus following gadobenate dimeglumine in patients with and without previous multiple administrations of gadodiamide. Eur Radiol 2016.

[31] Stojanov D, Aracki-Trenkic A, Benedeto-Stojanov D. Gadolinium deposition within the dentate nucleus and globus pallidus after repeated administrations of gadolinium-based contrast agents-current status. Neuroradiology 2016:1-9.

[32] Blakemore S-J. Imaging brain development: the adolescent brain. NeuroImage 2012;61(2):397-406 [Elsevier Inc.].

[33] Stein J, Schettler T, Wallinga D, Valenti M. In harm's way: toxic threats to child development. J Dev Behav Pediatr 2002;23(1 Suppl.):S13-22.

[34] Kanal E, Tweedle MF. Residual or retained gadolinium: practical implications for radiologists and our patients. Radiology 2015;275(3):630-4.

[35] Thomsen HS. T1 hyperintensity in the brain after multiple intravenous injections of gadolinium-based contrast agents. Acta Radiol 2016 